\title{
DOUBLE STAR ASTROMETRY WITH THE HIPPARCOS DATA
}

\author{
F. MIGNARD \\ Observatoire de la Côte d'Azur/CERGA, \\ Av.Copernic 06130 Grasse, France
}

\begin{abstract}
As we approach the final processing of the observations carried out by HIPPARCOS, in particular for the double and multiple stars, it is possible to provide reliable statistics on the number of such objects detected and on the quality of the relative and absolute astrometry and photometry. About 24000 stars have been recognized as non-single, including 11000 already known as double and multiple before the mission and 13000 discovered by Hipparcos. Also, a subset of 16000 stars among the 24000 have been successfully solved for their relative coordinates (position angle and separation) with an accuracy in the range of 3 to 30 mas, including 7000 new double stars. I outline in this paper the principle of the internal recognition procedure and present some statistics on the solution.
\end{abstract}

\section{Introduction}

The Hipparcos mission was primarily dedicated to the production of an astrometric catalogue for the position, proper motion and parallax of about 118000 stars, with a nominal accuracy of 2 mas and 2 mas/year. The data processing scheme was designed at an early level in the mission definition and was optimized for single stars. However the determination of the parallax of the double stars, in particular for the thousand or so orbital double stars, is of the utmost importance for the determination of the stellar masses. It was then compelling to account for theses complex sources in the data processing. This raised essentially two questions :

- How to be sure that the observed signal is that of a non single star? Although the Hipparcos Input Catalogue includes such information, it was soon recognized that it was too incomplete and that an independent recognition procedure had to be worked out. Scientifically this 
proved to be a real bonanza with the detection of thousands of new double stars.

- Once a program star is known to be double or multiple, it must be processed differently from the single stars because of lack of simple relationship between the phase of the signal and the position of the components on the sky. A by-product of this process is the measurement of the separation and position angle of about 16000 double stars, along with the difference of magnitude between the two components.

\section{Double star recognition}

Observations were carried out over essentially two continuous stretches from December 1989 to July 1992 and then from November 1992 up to March 1993 with a smaller daily coverage in the latter interval due to attitude recovery problems. Altogether this amounts to 37 months of data with a continuous flow of 24000 bits per seconds. On the average a particular star has been observed about 120 times over this period, with considerable variation from star to star with the ecliptic latitude.

The basic signal is described in Kovalevsky et al. (1992), and for double stars in Mignard et al. (1992). The expected signal is properly calibrated for a point source, it is then possible to study the deviation of the actual signal from this ideal and construct statistical tests to reject the hypothesis that the source is single. In particular, one of the tests is based on the photometry computed on the average intensity on one hand, and on the amplitude of modulation on the other hand. The two magnitudes so derived are equal for a single star, whereas the latter is larger than the former for a double star. A plot of the detections is shown in Fig. 1 for about 10000 stars as a function of the color. The diagram is very asymetrical, with virtually no negative values, but those accounted for by the photon noise. In the upper part of the diagram $(\Delta m>0)$ all the data points with a magnitude difference larger than 0.025 mag very likely correspond to non single stars, primarily doubles. In this plot one also notes that, thanks to a careful calibration, there is no systematic effect, even for the reddest stars. The statistics of detection are given in table 1, and are split into three categories. A star is categorized as single when the signal has never shown any significant departure from the single star signal over the 120 passages. On the other hand it is said to be double or multiple beyond a certain statistical threshold which rules out it being single. There remains an intermediary class for which no firm decision can be made with the data collected by Hipparcos. 


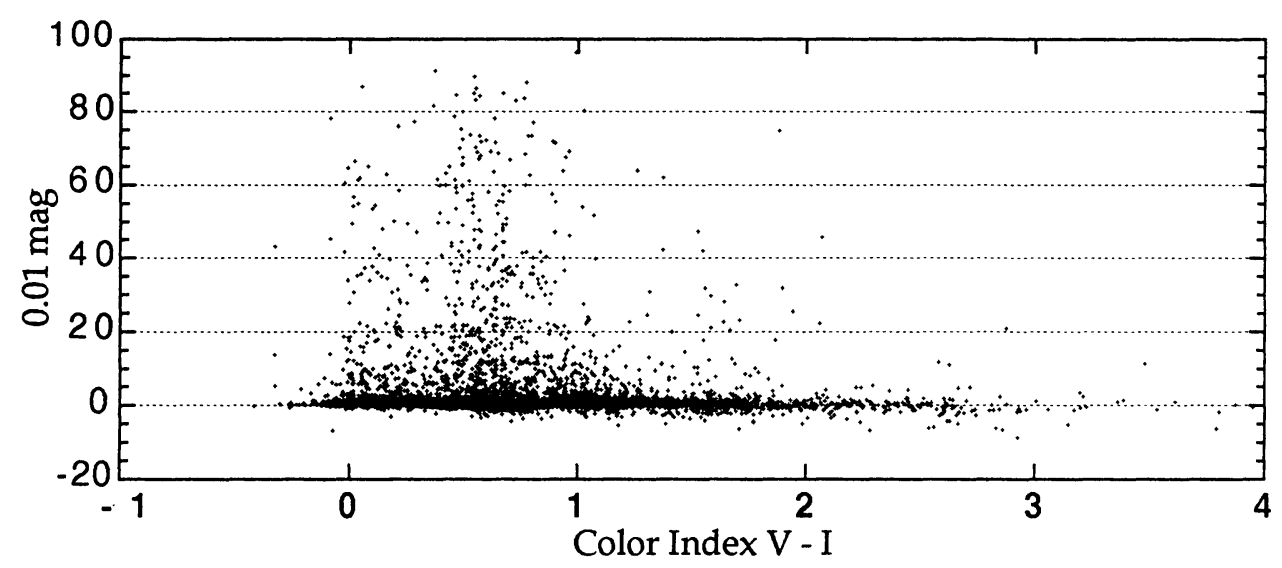

Figure 1. Photometric detection of double stars

TABLE 1. Double star detection

\begin{tabular}{ll}
\hline Category & N \\
\hline Single & 83000 \\
Dubious & 10500 \\
Double or multiple & 24500 \\
\hline Total & 118000 \\
\hline
\end{tabular}

\section{Double star solution}

\subsection{THE RELATIVE ASTROMETRY}

We cannot in this short paper describe the convoluted track that leads from the reception of the raw data to the astrometric solution. This has been described in several publications, for example in Kovalevsky et al. (1992), Perryman et al. (1992). In the case of double star a fitting of the grid signals, collected over all the available transits, to the double star parameters provides the separation, the position angle and the magnitude difference. Then at a latter stage one can find the absolute position and parallax.

One must stress however that not every double star is seen as such by Hipparcos, in the same way as there are detection limits in ground based observations. Regarding the small separations, the diffraction by the Hipparcos window sets the resolving power at $\rho>0.10-0.15^{\prime \prime}$. So stars with smaller separations are termed single, although they might be double, and 
actually quite often are. For these stars, close to the lower limit of resolution, the magnitude difference is strongly correlated to the separation, and in general cannot be large. On the other hand at the upper limit, the detector allows a detection of the companion, provided it is at a distance from the primary less than 25 to 30 arcsec. This instrumental limitation is due to the using of a image dissector tube (IDT) which diminishes the available light of an image not properly centered on the detector, in such a way that a star lying 20 arcsec off center appears 1.5 mag fainter, nearly 3 mag at $25 \operatorname{arcsec}$ and $6 \mathrm{mag}$ at 30 arcsec. For these stars the detection limit for the magnitude difference is about $\Delta m \approx 4 \mathrm{mag}$, including the attenuation effect. So a pair with a separation larger than 25 arcsec generates a signal hardly discernible from that of a single star and is categorized as single on the sole basis of Hipparcos observations.

The statistics of the solution are given in table 2 and refer to the solution before a full synthesis between NDAC and FAST is completed. The figures are then not final, but should be within $10 \%$ of the catalogue content. Table 2 refers to the 24500 stars considered non-single in Table 1 . A complete solution means that both the relative astrometry and photometry have been obtained from the transit data. It may seem that the solution is made more likely when a star is known to be double from pre-launch observations. This reflects the fact that the range of known visual binaries, which make most of the content of the Input Catalogue double stars, comprises stars rather easily solved with the Hipparcos observations, with separations larger than 0.2 arcsec and magnitude difference less than 3 mag. So out of the 11000 stars detected, about $90 \%$ are properly solved. The situation is very different for the new double stars, which are on the average of smaller separation and/or larger magnitude difference. So they are detected as non single from the signal, but it is more difficult to retrieve the two components.

TABLE 2. Double star solution statistics

\begin{tabular}{lccc}
\hline & Total & Known DS & New DS \\
\hline Complete Solution & 16500 & 9500 & 7000 \\
Photometry only & 8000 & 1500 & 6500 \\
\hline Total & 24500 & 11000 & 13500 \\
\hline
\end{tabular}

The distribution of the separations are given in Fig. 2 and 3, respectively for the known and new double stars. The difference is conspicuous, with the large fraction of close binaries belonging to the set of Hipparcos detected 


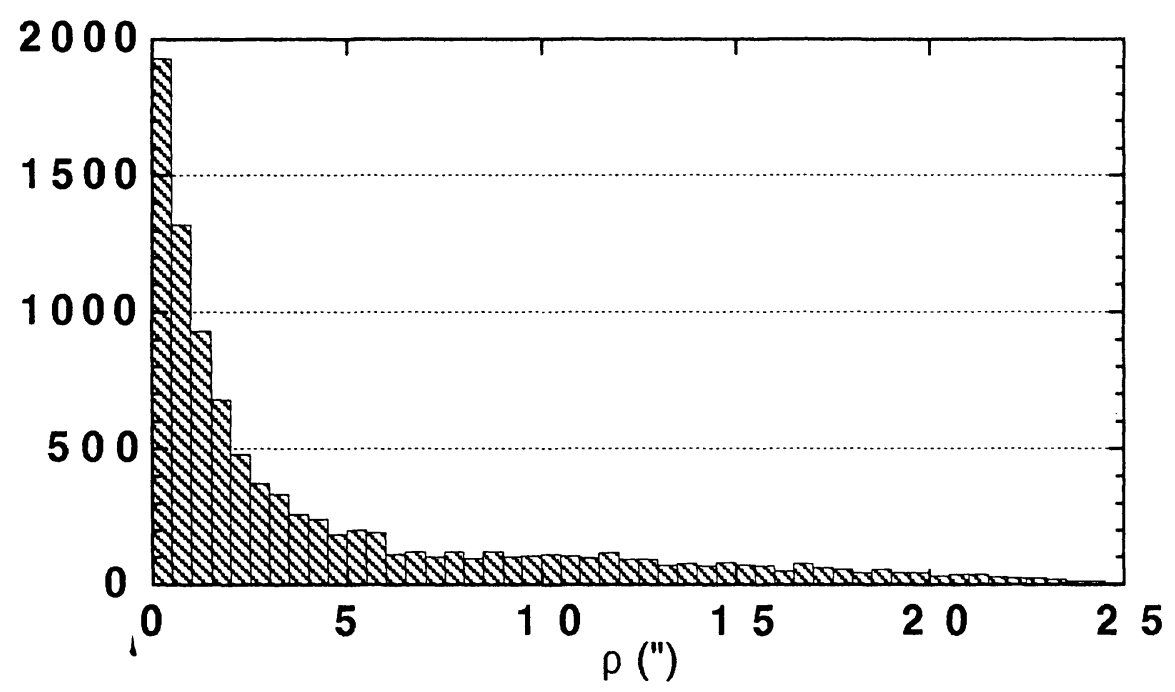

Figure 2. Distribution of the separation for 9000 known double stars

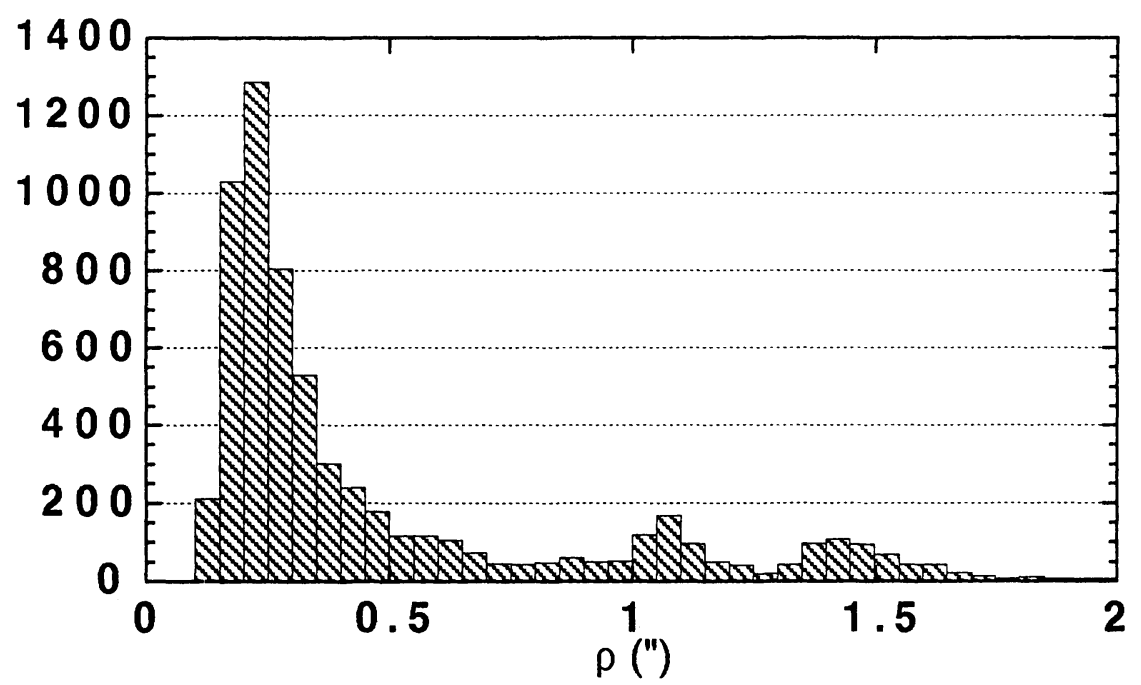

Figure 3. Distribution of the separation for 6000 new double stars

double stars. The two bumps at $\rho \approx 1.2 \operatorname{arcsec}$ and $\rho \approx 1.4$ arcsec are instrumental effects. The first is caused by grid step errors and is probably not real while the second illustrates a better detection efficiency at the separation of 1.4 arcsec.

Comparison to external data of similar accuracy can be made with the observations of double stars made by speckle interferometry and published 


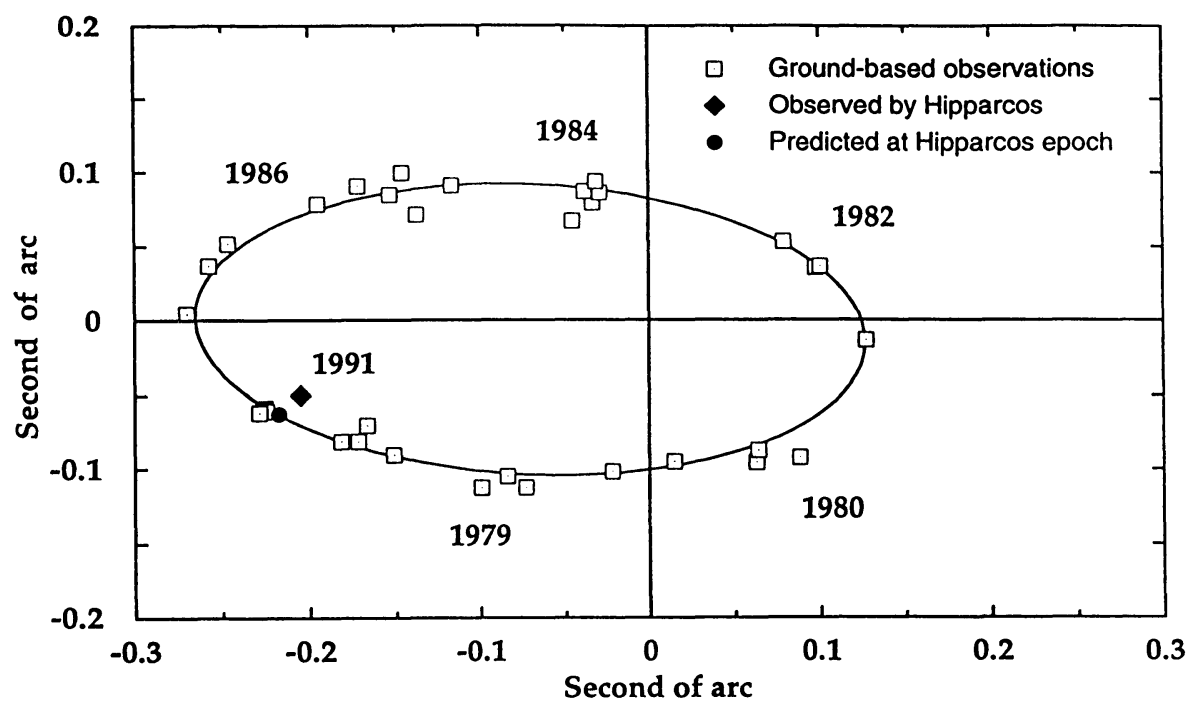

Figure 4. Comparison of an Hipparcos observation to ground based measurements

by McAlister and Hartkopf (1988). One such example is shown in Fig.4 for a star observed regularly over an orbit. The Hipparcos data point matches the predicted position within the expected accuracy of 12 mas on each direction for this object. From our analysis, it seems that the Hipparcos separation are free of systematic error above one mas, at least for separation larger than 0.2 arcsec. For smaller separation this is not yet fully known.

\subsection{THE ABSOLUTE ASTROMETRY}

The parameters of the double stars are then used to correct the signal from the effect brought about by the duplicity and solve for the absolute astrometry in the same way as for a single star. In an alternative approach used by the NDAC consortium, one fits in a single step both the relative and absolute astrometry and photometry to the grid signal. Thus the methods used by the two consortia differ markedly, to such an extent that the comparison of the results is really meaningful, since the systematic errors that may remain in the solutions are unlikely to be similar. This proved to be a very efficient tool to detect and correct shortcomings in either software. At the end the analysis of the scatter between the two solutions is probably a good indication of the external error. In no case can it supersede the comparison to fully external data, provided they are of comparable accuracy with Hipparcos.

The main result is illustrated in Fig. 5, with the difference in the parallax NDAC - FAST for about 3000 double stars solved by both groups and for 


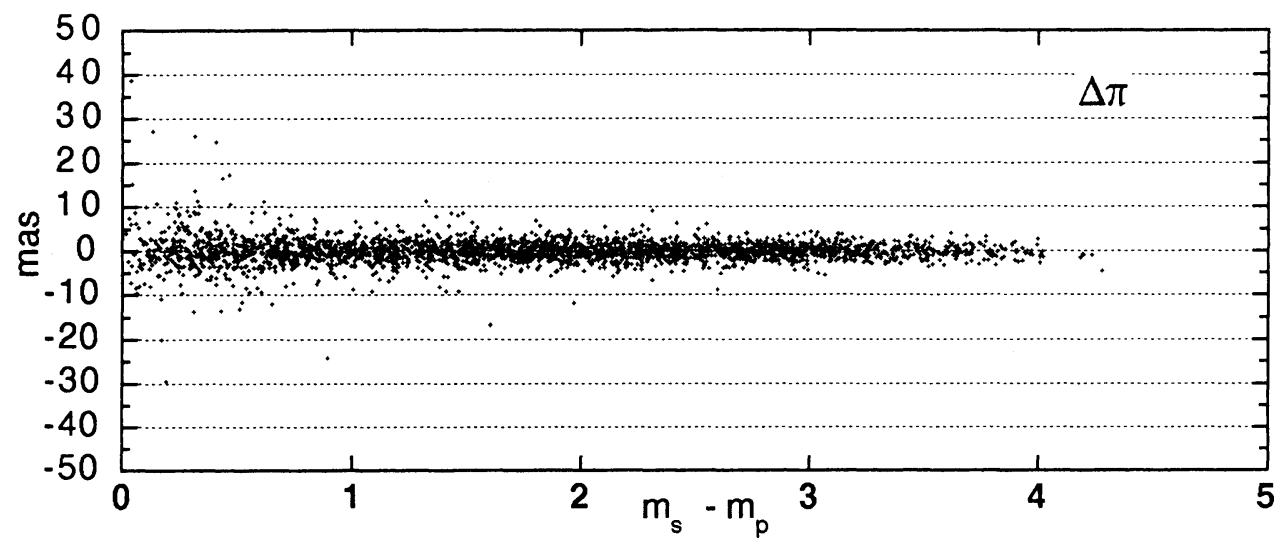

Figure 5. Parallax difference in mas between NDAC and FAST for 3000 double stars

which there is a good agreement in the solution found for the separation and position angle. In general the difference in the parallax is below 3.5 mas with a smaller scatter when the magnitude difference is large, that is to say when the single star conditions are recovered. This plot indicates the level of degradation in the parallax solution between the single stars, with a scatter of about 2 mas and the true double star, with a scatter about 1.5 times that of single stars.

One may fear that the astrometry might be very different in NDAC and FAST for stars processed as doubles by one group and as singles by the other. It turns out that such a situation occurs only for stars at the detection margins, that is to say with a signal not far from the single star signal. So the modeling error is not large on the astrometric parameters. More specifically those stars have usually very small separations and the position produced by Hipparcos will be that of the photocenter, which is exactly what we arrive at by neglecting the duplicity, provided $\rho<0.35$ arcsec. Experiments have shown that the effect on the parallax is then negligible.

\section{Conclusion}

The mass processing will continue within each consortium until mid 1995 to produce the final iterated solutions based on the whole data set, including all the single and multiple stars. Then the two independent solutions will be merged into the single Hipparcos catalogue both for the astrometry and photometry. 


\section{Acknowledgements}

The double star reduction of the Hipparcos data involves many scientists of different countries. They are gratefully acknowledged for their contribution : L. Lindegren, S. Söderhjelm (Sweden), J.L. Falin, M. Froeschlé, J. Kovalevsky, C. Martin (France), M. Badiali, P.L. Bernacca, L. Borriello,D. Cardini, R. Pannunzio, G. Prezioso, A. Spagna (Italy), H. Bernstein (Germany), P. Lampens (Belgium).

\section{References}

Kovalevsky J. et al. (1992) The FAST Hipparcos Data Reduction consortium: overview of the reduction software $A \mathscr{E} A$,Vol. no. 258 , pp. 7-17.

McAlister H.A. and Hartkopf W. I. (1988) Second Catalog of Interferometric Measurements of Binary Stars, Chara Contribution no. 2

Mignard F., Froeschlé M., Badiali M., Cardini D., Emanuele A.,Falin J.L., Kovalevsky J. (1992) Hipparcos double star recognition and processing within the FAST consortium, $A \mathscr{E} A$, Vol. no. 258 , pp. 165-172.

Perryman M.A.C. et al. (1992), In orbit performance of the Hipparcos astrometry satellite, $A \mathscr{E} A$, Vol. no. 258, pp. 1-6.

Turon C. et al. (1992), The Hipparcos Input Catalogue, ESA SP-1136. 